Pedro R Gil-Monte'

Mariana A Marucco"

\section{Prevalencia del "síndrome de quemarse por el trabajo" (burnout) en pediatras de hospitales generales}

\section{Burnout prevalence in pediatricians of general hospitals}

\section{RESUMEN}

OBJETIVO: Analizar la prevalencia del síndrome de quemarse por el trabajo (burnout) en pediatras de hospitales generales siguiendo criterios de diferentes países.

MÉTODOS: Estudio transversal, no aleatorio, realizado en Buenos Aires, Argentina, en 2006. La muestra la formaron 123 pediatras que trabajaban en Servicios de Pediatría de hospitales generales, 89 fueron mujeres $(72,4 \%)$ y 34 hombres $(27,6 \%)$. Los datos fueron recogidos mediante un cuestionario anónimo y autoadministrado. El SQT fue diagnosticado utilizando el Maslach Burnout Inventory, con diferentes criterios para establecer su prevalencia.

RESULTADOS: La prevalencia del SQT, varió en función del criterio utilizado: siguiendo los puntos de corte del manual de Estados Unidos, la prevalencia fue del 10,6\% de 24,4 \% siguiendo los criterios de España, 37,4\% siguiendo los criterios de Argentina y considerando los criterios clínicos establecidos en Holanda, el porcentaje fue del 3,2\%.

CONCLUSIONES: Los niveles de prevalencia variaron significativamente en función de los criterios aplicados debido a influencias transculturales.

DESCRIPTORES: Médicos Hospitalarios. Recursos Humanos, Hospital, Psicología. Síndrome de quemarse por el trabajo, clasificación. Evaluación del Rendimiento de Empleados. Satisfacción en el Trabajo. Estudios Transversales.
Unidad de Investigación Psicosocial de la Conducta Organizacional. Facultad de Psicología. Universidad de Valencia. Valencia, España

Servicio de Pediatría. Hospital Zonal General de Agudos Dr. A. Oñativia. Buenos Aires, Argentina 


\begin{abstract}
OBJECTIVE: To assess the prevalence of burnout in pediatricians of general hospitals.

METHODS: Non-randomized cross-sectional study carried out in the city of Buenos Aires, Argentina, in 2006. The study sample comprised 123 pediatricians working in pediatrics services of general hospitals, 89 women $(72.4 \%)$ and 34 men $(27.6 \%)$. Data were gathered through an anonymous, self-administered questionnaire. Burnout was measured using the Maslach Burnout Inventory, and different approaches were employed to estimate burnout prevalence.
\end{abstract}

RESULTS: The prevalence of burnout was different according to the approach used: the prevalence was $10.6 \%$ by the United States criteria; $24.4 \%$ by the Spanish criteria; $37.4 \%$ by the Argentinean criteria and $3.25 \%$ by the Dutch clinical criteria.

CONCLUSIONS: Burnout prevalences varied significantly depending on the approach used due to cross-cultural influences.

DESCRIPTORS: Hospitalists Personnel, Hospital, psychology. Burnout, Professional, classification. Employee Performance Appraisal. Job Satisfaction. Cross-Sectional Studies.

\title{
INTRODUCCIÓN
}

El síndrome de quemarse por el trabajo (SQT) (burnout) es una respuesta al estrés laboral crónico que aparece cuando fallan las estrategias de afrontamiento que habitualmente emplea el individuo para manejar los estresores laborales. El síndrome de quemarse por el trabajo se comporta como variable mediadora entre el estrés percibido y sus consecuencias (Gil-Monte ${ }^{4} 2005$ ). Ha sido definido como "un síndrome de agotamiento emocional, despersonalización y baja realización personal en el trabajo que puede desarrollarse en aquellos individuos que trabajan con personas en cualquier tipo de actividad (Maslach et $\mathrm{al}^{11}$ 2001). La baja de realización personal en el trabajo es una dimensión de carácter cognitivo consistente en una autoevaluación del individuo, definida como la tendencia de los profesionales a evaluarse negativamente. Esa evaluación negativa afecta de forma especial a la habilidad para la realización del trabajo y para relacionarse con las personas a las que atienden. El agotamiento emocional es un componente emocional que se define como la situación en la que los profesionales perciben que ya no pueden dar más de sí mismos a nivel afectivo. La despersonalización, es el desarrollo de actitudes y sentimientos negativos hacia las personas destinatarias del trabajo. Estas personas son vistas por los profesionales de manera deshumanizada debido a un endurecimiento afectivo, lo que conlleva que les culpen de sus problemas.

El diagnóstico y evaluación del síndrome de quemarse por el trabajo resulta de gran importancia para decidir cuándo, dónde, y sobre quién se debe intervenir; y para concluir sobre la prevalencia e incidencia del fenómeno. La realización de un diagnóstico adecuado requiere la evaluación de los síntomas del trabajador y la intensidad o frecuencia que presentan. La aparición del síndrome de quemarse por el trabajo en la literatura científica originó el desarrollo de una serie de instrumentos psicométricos dirigidos a su evaluación. El método más empleado para estimar la patología es el cuestionario, en detrimento de otros procedimientos como la observación clínica, la entrevista estructurada y las técnicas proyectivas.

La mayor incidencia de síndrome de quemarse por el trabajo se da en profesionales que ejercen una función asistencial o social, por lo que el deterioro de su calidad de vida laboral también conlleva repercusiones sobre la sociedad en general.

Los pediatras tienen la responsabilidad sobre la salud integral de niños y adolescentes. La relación médicopaciente-familia, habitualmente está enmarcada en circunstancias de extrema ansiedad y aflicción, con demanda de implicación emocional del pediatra en los problemas de los pacientes. El trabajo pediátrico presenta continuas interrupciones y reorganizaciones, y se ve condicionado por el exceso de ingresos por la vía no programada: "urgente", lo que lleva a la máxima ocupación de turnos y a la saturación del sistema. Esto motiva falta de control sobre el contenido y la plani- 
ficación del trabajo, a lo que se suma las restricciones presupuestarias con escasez de personal y recursos. La ausencia de autonomía para gestionar el trabajo y la formación, la falta de confianza de los pacientes, las interrupciones en el trabajo, el poco reconocimiento de los jefes, un sueldo escaso, un tiempo por enfermo aún más escaso, el exceso de demanda, la burocracia, la sensación de que la formación continua carece de sentido, ya que las posibilidades de promoción son muy escasas provocan: sobrecarga de trabajo, desmotivación, frustración y condicionan la vulnerabilidad del pediatra al síndrome de quemarse por el trabajo.

La mayoría de los estudios en los que se analiza la prevalencia del síndrome de quemarse por el trabajo e han realizado con el Maslach Burnout Inventory (MBI) (Maslach \& Jackson ${ }^{10}$ 1986). La aceptación y la difusión de este cuestionario han permitido realizar estudios en diferentes países y comparar los resultados obtenidos. Maslach \& Jackson ${ }^{10}$ (1986) proponen un criterio de percentiles para cada dimensión, consistente en dividir a la muestra en tres grupos de igual tamaño, $33,3 \%$ de los individuos. Estos grupos catalogan a las personas que responden el cuestionario en individuos con niveles altos, medios y bajos para cada dimensión (agotamiento emocional, despersonalización o realización personal en el trabajo). Esta manera de proceder entraña sesgos que pueden ser relevantes. Se debe tener en cuenta que los criterios estadounidenses pueden ser inadecuados en otros países, debido a la existencia de diferencias significativas en los valores normativos de corte, por cuestiones socioculturales. Esto dificulta concluir sobre el número de individuos que han desarrollado la patología y los que no (Gil-Monte \& Peiró ${ }^{6}$ 2000). En el manual no se ofrecen puntos de corte o criterios diagnósticos "validados clínicamente".

En España, Gil-Monte ${ }^{5}$ (2005) ha realizado la validación factorial del Maslach Burnout Inventory versión Human Services Survey (MBI-HSS). Así mismo estableció los valores medios en las tres subescalas del MBI obteniendo para la realización personal en el trabajo y el agotamiento emocional valores más altos que Maslach \& Jackson ${ }^{10}$ (1986), y para despersonalización valores más bajos (Gil-Monte \& Peiró ${ }^{6}$ 2000). En Argentina, $\mathrm{Neira}^{12}$ (2004) ha realizado la validación del MBIHSS en profesionales de la salud. Los profesionales argentinos obtuvieron en las escalas agotamiento emocional y despersonalización del MBI-HSS, una media aritmética más baja que el grupo sanitario estudiado en Estados Unidos (Maslach \& Jackson ${ }^{10}$ 1986). En la escala realización personal en el trabajo la media fue similar. Al calcular el puntaje promedio obtenido por los participantes en las escalas del MBI, observó que el agotamiento emocional y la despersonalización eran más altos en los médicos que en las enfermeras y otros profesionales sanitarios.

Otro criterio para determinar la prevalencia de síndrome de quemarse por el trabajo es el de Schaufeli \& Dierendonck $^{13}$ (1995) quienes establecieron puntos de corte para el MBI que se consideran validados clínicamente. El trabajo fue realizado en una muestra de trabajadores de la salud encaminados para tratamiento psicopatológico por problemas de salud mental derivados del trabajo. Estos autores recomiendan utilizar estos criterios para establecer el diagnóstico del síndrome de quemarse por el trabajo para evitar considerar a individuos sanos como enfermos.

La prevalencia del síndrome de quemarse por el trabajo en pediatras ha sido poco estudiada. La literatura ofrece diferentes estudios en los cuales pediatras forman parte de la muestra, en los que se ha aplicado el MBI-HSS con versiones más cortas, o con un rango de la escala Likert diferente al del manual (Bustinza et $\mathrm{al}^{1}{ }^{2000}$; Jiménez Álvarez et al ${ }^{7}$ 2002). Algunos estudios establecen puntos de corte propios, la mayoría de las veces no fundamentado o definido previamente, o suman las puntuaciones obtenidas en las tres subescalas y a partir de allí establecen las categorías alto, medio y bajo, por lo que se dificulta la posibilidad de establecer comparaciones. En un estudio realizado en España en personal asistencial pediátrico, López Franco et $\mathrm{al}^{9}$ (2005) obtuvieron una prevalencia del 20,8\%. Otro estudio de Jiménez Álvarez et al $^{7}$ (2002) en cirujanos pediatras obtuvo una prevalencia del 5,9\%, mientras que Bustinza et $\mathrm{al}^{1}$ (2000) en una muestra de pediatras intensivistas $(\mathrm{N}=49)$ observó una prevalencia del 10\%. En Brasil, Dutra (2004)a observó una prevalencia del $53,7 \%$ en un estudio realizado a pediatras en un hospital universitario $(\mathrm{N}=41)$ utilizando los puntos de corte del manual de Estados Unidos.

Dado que en Argentina estudios sobre la prevalencia del síndrome de quemarse por el trabajo en pediatras no existen, y a nivel internacional son escasos, el objetivo del presente estudio fue analizar la prevalencia en hospitales generales del sur del conurbano bonaerense.

\section{MÉTODOS}

Los servicios de pediatría de los hospitales estudiados en el conurbano bonaerense se encuentran localizados en zonas consideradas de riesgo epidemiológico. Las dos terceras partes de los bonaerenses habitan en el Conurbano, el cual representa la mayor concentración de áreas de pobreza de la Provincia de Buenos Aires. La región sanitaria estudiada ha presentado tasas de

${ }^{a}$ Dutra F. Características da incidência de burnout em pediatras de uma organização hospitalar pública. [dissertação mestrado]. Florianópolis: Centro de Filosofia e Ciências Humanas da Universidade Federal de Santa Catarina; 2004. Disponible en URL: http://www.tede.ufsc.br/teses/ PPSI0135.pdf [2007 agosto 7]. 
mortalidad infantil de $13,9 \%$, la pobreza es del $44,4 \%$ y la indigencia del 16,9\%. Debido al peso demográfico de la región los pobres se traducen en 4,3 millones y 1,65 millones de indigentes, según datos del Instituto Nacional de Estadística y Censo de Argentina correspondientes a 2001 .

La muestra quedó conformada por 123 pediatras. La tasa de respuesta fue del 53,9\%, considerando como población todos los pediatras con trabajo estable (de planta y guardia) de los hospitales generales de una región sanitaria del conurbano bonaerense. Trenta y tres participantes $(26,8 \%)$ trabajaban como personal de planta de 36 horas semanales (no realizan turno) y 90 participantes $(73,2 \%)$ tenían nombramiento de turno (24 horas de turno y 12 horas en planta). La distribución según sexo fue de 89 mujeres $(72,4 \%)$ y hombres 34 hombres $(27,6 \%)$. La media de edad ha sido de 42,4 años (24 -70 años).

El diseño fue transversal y no aleatorizado. Para la recogida de la información, se utilizó un cuestionario anónimo y autoadministrado. Se obtuvo respuesta en todos los hospitales.

El síndrome de quemarse por el trabajo se estimó mediante la adaptación al español (Gil-Monte ${ }^{5}$ 2005) del MBI-HSS (Maslach \& Jackson ${ }^{10}$ 1986). El cuestionario consta de 22 ítems que según señala el manual se distribuyen en tres escalas denominadas: realización personal en el trabajo ( 8 ítems, $\alpha=0.84$ ), agotamiento emocional ( 9 ítems, $\alpha=0.88$ ) y despersonalización ( 5 ítems, $\alpha=0.78$ ). Los individuos valoran cada ítem del cuestionario con una escala tipo Likert en la que indican la frecuencia con la que han experimentado la situación descrita en el ítem durante el último año. Esta escala de frecuencia tiene siete grados que van de cero ("nunca") a seis ("todos los días"). Bajas puntuaciones en realización personal en el trabajo junto a altas puntuaciones en agotamiento emocional y en despersonalización se corresponden con altos niveles del síndrome de quemarse por el trabajo.

Para establecer criterios de riesgo se utilizaron los puntos de corte de Maslach \& Jackson ${ }^{10}$ (1986), los de Gil-Monte \& Peiró $^{6}$ (2000) y los de Neira ${ }^{12}$ (2004). Para establecer criterios patológicos se utilizaron los puntos de corte de Schaufeli \& Dierendock ${ }^{13}$ (1995).

Para el análisis estadístico se utilizó el programa SPSS 11.0.

Se solicitaron los consentimientos informados a los respectivos directores, jefes de servicio, comités de ética, y comités de docencia e investigación de cada hospital, así como un consentimiento a cada participante, que fue entregado en hoja aparte.

\section{RESULTADOS}

Como se puede observar en la Tabla, siguiendo los puntos de corte establecidos por Maslach \& Jackson ${ }^{10}$ (1986), 42 participantes $(34,1 \%)$ puntuaban bajo en realización personal en el trabajo, $58(47,1 \%)$ puntuaron alto en agotamiento emocional, y $28(22,8 \%)$ puntuaron alto en despersonalización. Aplicando los criterios de Gil-Monte \& Peiró 6 (2000), esos valores fueron respectivamente: 73 participantes $(59,3 \%), 64(52,0 \%) \mathrm{y}$ 47 (38,2\%); utilizando los puntos de corte de Neira ${ }^{12}$ (2004): 73 (59,3\%), 70 (56,9\%) y 73 (59,3\%). Según los puntos de corte establecidos por Schaufeli \& Dieren$\operatorname{donck}^{13}$ (1995) se obtuvo 23 participantes $(18,7 \%)$ con baja realización personal en el trabajo, 24 (19,5\%) altos niveles de agotamiento emocional, y 30 participantes $(24,4 \%)$ con altos niveles de despersonalización.

Como se aprecia en la Figura 1, los niveles de baja realización personal en el trabajo fueron similares aplicando los criterios de Gil-Monte \& Peiró ${ }^{6}$ (2000),

Tabla. Prevalencia del síndrome de quemarse por el trabajo según puntos de corte y porcentaje de individuos afectados. Buenos Aires, Argentina, 2006.

\begin{tabular}{|c|c|c|c|c|c|c|c|c|c|c|c|c|}
\hline \multirow{2}{*}{$\begin{array}{l}\text { Puntos } \\
\text { de corte }\end{array}$} & \multicolumn{3}{|c|}{$\begin{array}{c}\text { Maslach \& } \\
\text { Jackson" }{ }^{11}(1986)\end{array}$} & \multicolumn{3}{|c|}{ Gil-Monte \& Peiró 7 (2000) } & \multicolumn{3}{|c|}{ Neira $^{13}(2004)$} & \multicolumn{3}{|c|}{$\begin{array}{c}\text { Schaufeli \& } \\
\text { Dierendonck }^{14} \text { (1995) }\end{array}$} \\
\hline & RPT & $\mathrm{AE}$ & DEP & RPT & $\mathrm{AE}$ & DEP & RPT & $\mathrm{AE}$ & DEP & RPT & $\mathrm{AE}$ & DEP \\
\hline Alto & $\geq 39$ & $\geq 27$ & $\geq 13$ & $\geq 40$ & $\geq 25$ & $\geq 9$ & $\geq 41$ & $\geq 22$ & $\geq 5$ & $\geq 29$ & $\geq 34$ & $\geq 12$ \\
\hline$(\%)$ & $(27,6)$ & $(47,1)$ & $(22,8)$ & $(26,8)$ & $(52,0)$ & $(38,2)$ & $(23,6)$ & $(56,9)$ & $(59,3)$ & $(75,6)$ & $(19,5)$ & $(24,4)$ \\
\hline Medio & $38-32$ & $26-17$ & $12-7$ & $39-36$ & $24-16$ & $8-4$ & $36-40$ & $12-21$ & $3-6$ & $28-26$ & $33-26$ & $11-6$ \\
\hline$(\%)$ & $(38,2)$ & $(23,6)$ & $(30,9)$ & $(13,8)$ & $(22,8)$ & $(36,6)$ & $(17,1)$ & $(29,7)$ & $(21,9)$ & $(5,7)$ & $(30,9)$ & $(34,9)$ \\
\hline Bajo & $\leq 31$ & $\leq 16$ & $\leq 6$ & $\leq 35$ & $\leq 15$ & $\leq 3$ & $\leq 35$ & $\leq 11$ & $\leq 2$ & $\leq 25$ & $\leq 25$ & $\leq 5$ \\
\hline$(\%)$ & $(34,1)$ & $(29,3)$ & $(46,3)$ & $(59,3)$ & $(25,2)$ & $(25,2)$ & $(59,3)$ & $(13,8)$ & $(18,7)$ & $(18,7)$ & $(49,6)$ & $(40,7)$ \\
\hline \% SQT & & $10,6 \%$ & & & $24,4 \%$ & & & $37,4 \%$ & & & $3,2 \%$ & \\
\hline
\end{tabular}

RPT: realización personal en el trabajo

AE: agotamiento emocional

DEP: despersonalización

SQT: síndrome de quemarse por el trabajo 


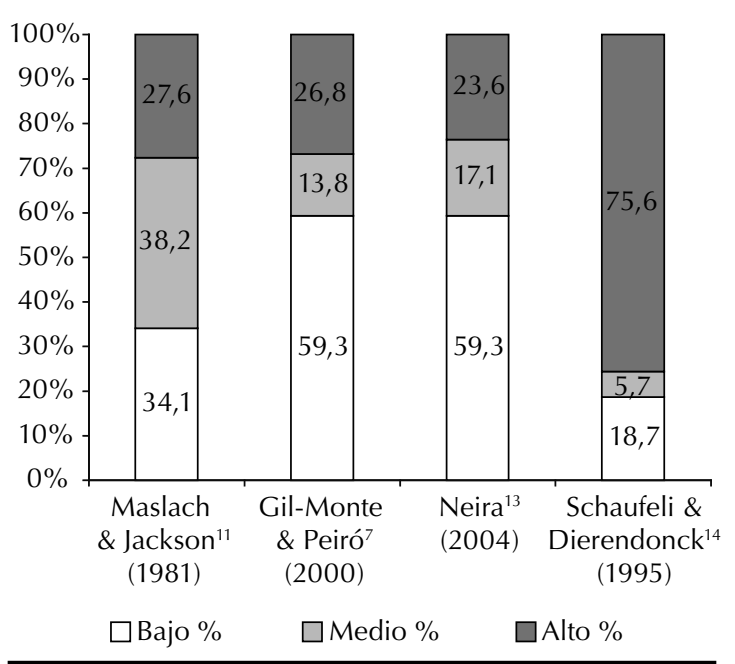

$\overline{\text { Figura 1. Prevalencia de realización personal en el trabajo en }}$ la muestra del estudio aplicando diferentes criterios. Buenos Aires, Argentina, 2006.

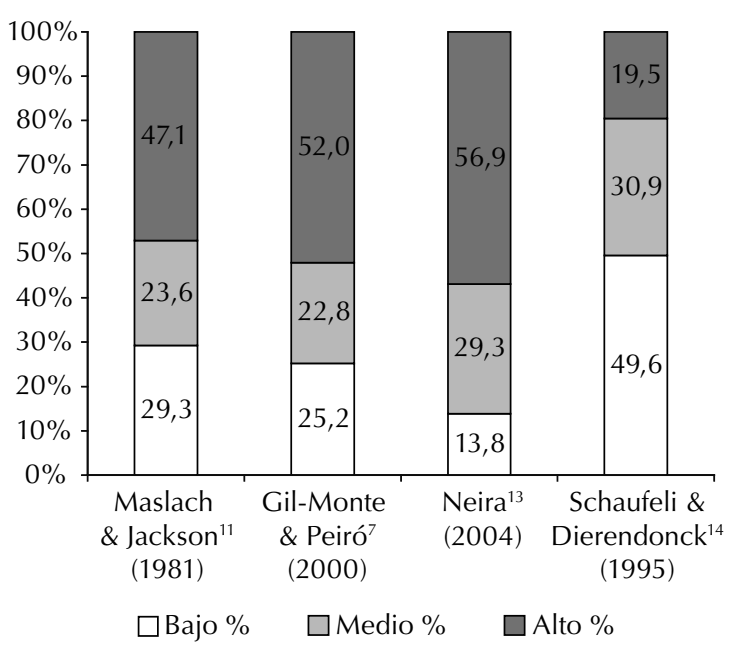

$\overline{\text { Figura 2. Prevalencia de agotamiento emocional en la mues- }}$ tra del estudio aplicando diferentes criterios. Buenos Aires, Argentina, 2006.

y de Neira ${ }^{12}$ (2004). Con los criterios de Maslach \& Jackson $^{10}(1986)$ resultaron inferiores, y aún menores al aplicar los puntos considerados por Schaufeli \& Dierendonck ${ }^{13}$ (1995) como puntos de corte clínico.

Cuando se compararon los niveles de agotamiento emocional (Figura 2), se observó que los niveles de prevalencia más elevados se correspondían con los puntos de corte establecidos por Neira ${ }^{12}$ (2004), resultando más bajos con los puntos de corte establecidos por Gil-Monte \& Peiró ${ }^{6}$ (2000), y aún menores con los de Maslach \& Jackson ${ }^{10}$ (1986). Como era de esperar los niveles de prevalencia más bajos en esta dimensión se correspondieron con los puntos de corte clínicos (Schaufeli \& Dierendonck ${ }^{13}$ 1995).

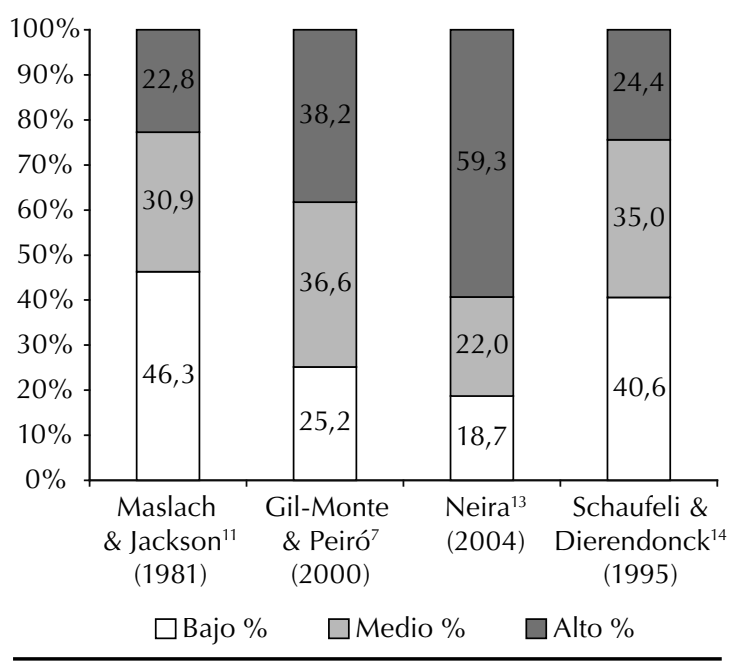

Figura 3. Prevalencia de despersonalización en la muestra del estudio aplicando diferentes criterios. Buenos Aires, Argentina 2006.

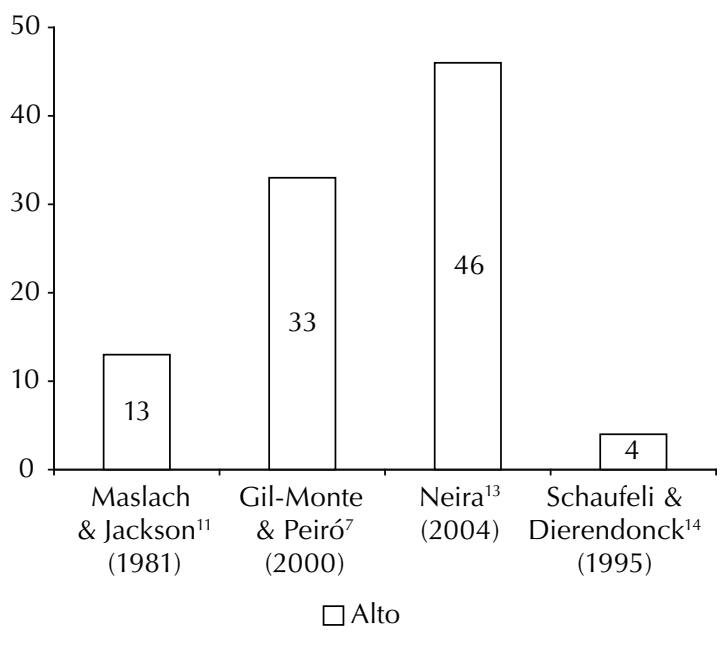

Figura 4. Prevalencia del síndrome de quemarse por el trabajo en la muestra del estudio aplicando diferentes criterios. Buenos Aires, Argentina, 2006.

Por lo que se refiere a los niveles de prevalencia obtenidos para despersonalización, los resultados comparativos fueron algo diferentes a los obtenidos para las otras dos dimensiones, pues la prevalencia fue superior aplicando los puntos de corte de Schaufeli \& Dierendock $^{13}$ (1995) que con los obtenidos por Maslach $\& \operatorname{Jackson}^{10}$ (1986) en el manual (Figura 3).

Un paso más en el estudio de la prevalencia del síndrome de quemarse por el trabajo consistió en analizar cuantos participantes de la muestra presentaban el síndrome. Para ello se consideró que los participantes debían cumplir el criterio de puntuar bajo en realización personal en el trabajo al tiempo que puntuar alto en agotamiento emocional y en despersonalización. En la 
Figura 4 se presentan los resultados comparativos según los diferentes criterios. Sólo 13 participantes (10,6\%) presentarían el síndrome de quemarse por el trabajo si se aplicaran los puntos de corte de Maslach \& Jackson ${ }^{10}$ (1986), mientras que la prevalencia se elevó a 33 participantes $(24,4 \%)$ con los criterios de Gil-Monte \& Peiró ${ }^{6}$ (2000), y a 46 participantes $(37,4 \%)$ con los criterios de Neira ${ }^{12}$ (2004). Por el contrario, la prevalencia del síndrome de quemarse por el trabajo resultó inferior cuando se utilizaron criterio clínicos para establecerla (Schaufeli \& Dierendonck $\left.{ }^{13} 1995\right)$, pues sólo 4 participantes $(3,2 \%)$ puntuaron bajo en realización personal en el trabajo, y al mismo tiempo alto en agotamiento emocional y en despersonalización.

\section{DISCUSIÓN}

Si bien la tasa de respuesta no fue uniforme (del 15\% al $100 \%$ ), los hospitales no presentan diferencias entre sí en lo que respecta a categoría profesional de los pediatras y su distribución por turno. La tasa de respuesta fue similar o superior a la obtenida en algunos estudios sobre síndrome de quemarse por el trabajo en personal sanitario (De Pablo González \& Superviola González ${ }^{3}$ 1998, Caballero Martín et $\mathrm{al}^{2}$ 2001), y similar a los estudios realizados en personal sanitario que trabaja con niños (Bustinza et $\mathrm{al}^{1}$ 2000, Jiménez et $\mathrm{al}^{7}$ 2002).

El estudio presenta algunas limitaciones. Al tratarse de cuestionarios anónimos, desconocemos las causas de no respuesta de algunos individuos. No se descarta la falta de motivación de la persona de referencia para realizar el estudio, en algunos centros asistenciales. Otra limitación del estudio es que el muestreo no fue aleatorio. Aunque en tres hospitales respondieron el 100\% de los pediatras, la tasa de prevalencia puede estar afectada por falsos positivos ("efecto del trabajador sano") (Karasek $\&$ Theorell $^{8}$ 1990). El resultado es que probablemente los valores normativos están sesgados, de tal forma que se concluye que determinados individuos presentan un alto grado en el síndrome de quemarse por el trabajo cuando realmente no es así. También se debe considerar la posibilidad de que las personas menos satisfechas con su calidad de vida profesional no hubieran contestado la encuesta. No obstante, la muestra quedó conformada por 89 mujeres $(72,4 \%)$ y 34 varones $(27,6 \%)$. Esta distribución puede ser considerada similar a la del colectivo de profesionales de pediatría en su conjunto y, junto a la tasa de respuesta obtenida y su comparación con otros estudios, le confiere validez externa a los resultados del estudio.

Uno de los principales aportes del presente estudio es la comparación de tasas de prevalencia siguiendo diferentes criterios normativos. La tasa de prevalencia obtenida con las puntuaciones de este estudio difiere de las obtenidas en las muestras normativas de Estados Unidos, España y Holanda. La falta de resultados trans- culturales que expliquen estas diferencias, sólo nos permite especular sobre su origen: significado del trabajo, las diferencias socio-económico-culturales entre América Latina - específicamente Argentina con la cultura norteamericana y europea -, o el rol del pediatra en la sociedad argentina afectado por el alejamiento del Estado de su responsabilidad de custodiar el bien común. Esta situación ha influido sobre el campo de acción de la pediatría y de sus principios éticos. Los pediatras tienen la responsabilidad sobre la salud integral de niños, pero los nuevos recursos conceptuales y tecnológicos para el cuidado y reparación de la salud frecuentemente no llegan a quienes más los necesitan. También resulta difícil lograr que los programas de perfeccionamiento puedan ser llevados efectivamente a la práctica. Esto ha generado que los pediatras se acostumbren y naturalicen los hechos circundantes, en situaciones de extrema pobreza. En Argentina se ha desarrollado de manera progresiva un deterioro creciente de las condiciones del ejercicio profesional de la pediatría. Esto interfiere en la relación medico-paciente y afecta la dignidad del trabajo pediátrico, trastocando así los fundamentos mismos de la medicina entendida como el enfoque solidario de la salud de una comunidad.

La prevalencia del presente estudio resultó aumentada al compararla con los resultados obtenidos por Nei$\mathrm{ra}^{12}$ (2004) en Argentina, en una muestra de médicos de diferentes especialidades, incluidos pediatras. Al aplicar los percentiles obtenidos por Neira ${ }^{12}$ (2004) a las puntuaciones de la muestra del presente estudio se obtuvo que el $39,4 \%$ de los pediatras presentaban el síndrome de quemarse por el trabajo, mientras que en la muestra de Neira fue del 14,6\%. La prevalencia del presente estudio también resultó más aumentada que la informada en otros estudios realizados con personal asistencial pediátrico $\left(20,8 \%\right.$ ) (López Franco et $\mathrm{al}^{9}$ 2005), o con pediatras de tratamiento intensivo (10\%) (Bustinza et $\mathrm{al}^{1}$ 2000). Sin embargo, esos estudios no se realizaron con los puntos de corte establecidos para el presente estudio, y/o utilizaron versiones del MBI-HSS acortadas o modificadas, lo que dificulta la comparación y establecer conclusiones. Este problema es relativamente frecuente en los estudios sobre prevalencia del síndrome de quemarse por el trabajo, pues el manual (Maslach \& Jackson ${ }^{10}$ 1986) aconseja dividir la muestra en tres grupos de similar tamaño siguiendo un criterio de percentiles $(33,3 \%$ de las puntuaciones en cada grupo).

Aunque en el manual se ofrecen valores normativos de referencia para los percentiles, no se presentan criterios para concluir cuando un individuo ha desarrollado la patología. Esta manera de proceder entraña sesgos que pueden ser relevantes, en especial cuando las puntuaciones obtenidas en los estudios no presentan una distribución normal, y están afectadas por una asimetría positiva. Por otra parte, se debe tener en cuenta que los 
criterios de unos países pueden ser inadecuados en otros debido a la existencia de diferencias significativas en los valores normativos de corte por cuestiones socioculturales (Gil-Monte \& Peiró ${ }^{6}$ 2000). Sería necesario desarrollar más estudios comparativos de este tipo en diferentes países de Latinoamérica para delimitar la dimensión del problema.

Como conclusión se considera que la prevalencia del síndrome de quemarse por el trabajo de los pediatras en el estudio es del 3,2\%. Tomando en cuenta consideraciones psicométricas, en estos casos se podría hablar de casos clínicos. Pero, esta conclusión está vinculada a la necesidad de realizar una entrevista clínica para

\section{REFERENCIAS}

1. Bustinza A, López-Herce J, Carrillo A, Vigil MD, de Lucas N, Panadero E. Situación de burnout de los pediatras intensivistas españoles. An Esp Pediatr. 2000;52(5):418-23.

2. Caballero Martín M, Bermejo Fernández F, Nieto Gómez R, Caballero Martínez F. Prevalencia y factores asociados al burnout en un área de salud. Aten Primaria. 2001;27(5):313-7.

3. De Pablo González R, Superviola González JF. Prevalencia del síndrome de burnout o desgaste profesional en los médicos de atención primaria. Aten Primaria. 1998;22(9):580-4.

4. Gil-Monte PR. El Síndrome de quemarse por el trabajo (burnout). Una enfermedad laboral en la sociedad de bienestar. Madrid: Pirámide; 2005.

5. Gil-Monte PR. Factorial validity of the Maslach Burnout Inventory (MBI-HSS) among Spanish professionals. Rev Saude Publica. 2005;39(1):1-8.

6. Gil-Monte PR, Peiró Silla JM. Un estudio comparativo sobre criterios normativos y diferenciales para el diagnóstico del síndrome de quemarse por el trabajo (Burnout) según el MBI-HSS en España. Rev Psicol Trab Org. 2000;16(2):135-49. confirmar el diagnóstico y descartar otros problemas que pueden influir en los síntomas evaluados, así como analizar la intensidad de las consecuencias del SQT en estos sujetos, y el grado de incapacidad que presentan para el ejercicio de su actividad laboral. Aún así, estos valores, junto al porcentaje de participantes que se consideran de riesgo hacen suponer un deterioro importante en la atención pediátrica ofrecida a la población. Por ello es necesario que los departamentos responsables de la atención sanitaria a la población y, en especial, los responsables de la atención pediátrica desarrollen una política de prevención e intervención para la disminución de los riesgos psicosociales que afectan a este colectivo ocupacional.
7. Jiménez Alvarez C, Morales Torres JL, Martínez Martínez C. Estudio del síndrome de "Burnout" en cirujanos pediatras españoles. Cir Pediatr. 2002;15(2):73-8.

8. Karasek R, Theorell T. Healthy work: Stress, productivity and the reconstruction of working life. Nova York: Basic Books; 1990.

9. López Franco M, Rodríguez Núñes A, Fernández Sanmartín M, Marcos Alonso S, Martinón Torres F, Martinón Sánchez JM. Síndrome de desgaste profesional en el personal asistencial pediátrico. An Esp Pediatr. 2005;62(3):248-51.

10. Maslach C, Jackson SE. Maslach Burnout inventory manual. 2. ed. Palo Alto: Consulting Psychologists Press; 1986.

11. Maslach C, Schaufeli WB, Leiter M. Job burnout. Annu Rev Psychol. 2001;52:397-422.

12. Neira C. Cuando se enferman los que curan. Estrés laboral y burnout en los profesionales de la salud. Buenos Aires: Gambacop; 2004.

13. Schaufeli WB, Van Dierendonck D. A cautionary note about the cross-national and clinical validity of cut-off points of the Maslach Burnout Inventory. Psychol Rep. 1995;76(2 Pt 2):1083-90.

Artigo basado en la tesis de doctorado de MA Marucco, presentada en la Universidad Nacional de Lanús (Buenos Aires, Argentina), en 2006.

Estudio presentado en el 34. Congreso Argentino de Pediatría, Ciudad de Córdoba (Argentina) en Octubre, 2006. 